Research Article

\title{
Spectrochemical Technology in Nanomaterial Preparation and Art Appraisal Technology Research
}

\author{
Xiao Tang (iD \\ Department of Oil Painting, Hubei Institute of Fine Arts, Wuhan 430205, Hubei, China \\ Correspondence should be addressed to Xiao Tang; tangxiao@hifa.edu.cn
}

Received 7 August 2020; Revised 4 September 2020; Accepted 20 September 2020; Published 15 October 2020

Academic Editor: Tifeng Jiao

Copyright (C) 2020 Xiao Tang. This is an open access article distributed under the Creative Commons Attribution License, which permits unrestricted use, distribution, and reproduction in any medium, provided the original work is properly cited.

With the rapid development of science and technology in micro/nanofield, it is urgent to carry out the research on new technology of micro/nanomechanics test and multiscale-related experimental mechanical analysis method. There is still a lot of work to be done in the basic theory and analysis method of micro-Raman spectroscopy as a new microscale mechanical test method applied to the experimental research of material mechanics. Based on the above background, the purpose of this paper is the application of spectrochemical technology in the preparation of nanomaterials and art identification technology. In this paper, different morphologies of $\mathrm{SiO}_{2}$ nanomaterials, such as crystalline nanoparticles, amorphous nanowires, nanospheres, and nanonets, were prepared by a simple hot steaming method in the presence of argon. The samples were characterized by Raman spectroscopy. In addition, this paper also carried out art identification work, using spectral technology to detect some paintings and calligraphy. The experimental results show that the Raman spectra of these paintings and calligraphy papers are in the visible region because their fluorescence is too strong, and almost all the peaks are submerged. In the near-infrared region (1064 nm), their peaks are also very weak, but they can be distinguished by fluorescence and scanning electron microscopy.

\section{Introduction}

The rapid development of micro-nanotechnology brings new challenges to machine experiments. Small-scale, largescale effect, multiscene combination effect, and nonclassical force become the factors to be considered, which is difficult to apply to many macroscopic high-type mechanical experiment methods. On the contrary, in the fine structure of materials, the study of macromechanical properties requires a variety of experimental tests and analysis. Therefore, it is very important to study the new testing technology and multifunctional correlation analysis method of micro/ nanomachinery. Raman spectroscopy is a new microexperimental mechanical testing technology developed recently. The basis of physical measurement is Raman spawning, which reflects the vibration energy information of material lattice, and the basic principle is determined deformation. The deformation rate is related to the fine mesh deformation, which can induce the change in Raman characteristic peak frequency, and the deformation (or stress) can be measured by sensing the change in Raman spectrum. This technology has the characteristics of noncontact, destruction, and high-spatial decomposition and can measure the inherent stress and external stress. It is a potential test method in the field of micromechanics characterization.

Template synthesis is an important kind of nanofabrication methods. Quintana reviewed the recent progress in the preparation of nanomaterials by nanoporous membrane extrusion, namely, "nonsacrificial template synthesis." Firstly, the types of nanoporous membranes in extrusion applications were introduced. Secondly, four common extrusion strategies of nanoporous membrane were studied: vesicle extrusion, membrane emulsification, precipitation extrusion, and biofilm extrusion. This paper discusses the principle and historical background of each specific technology, lists prominent examples, and evaluates their positive and negative characteristics. Finally, the research status and development prospect of nanoporous membrane extrusion methods are discussed. Abdel Salam 
studied the structure function relationship of new nanomaterials and its effect on the properties of concrete. In addition, Abdel Salam also discussed the mechanism of using new nanomaterials to improve the durability of concrete. Finally, the application of this material in practical engineering is briefly introduced. Gonciarz reported the preparation of nanosized cobalt disulfide by a simple liquidphase method with thiophenol as the end-capped agent in the cationic surfactant cetyltrimethylammonium bromide (CTAB) micelles. The product was characterized by spectrum, electron microscope, and magnetic moment measurement [1]. Duan first synthesized a novel multifunctional nanomaterial by combining superparamagnetism of nanoiron oxide with fluorescence properties of morin- $\mathrm{Al}_{3}{ }^{+}$ complex. First, the iron oxide nanoparticles are coated with silica to isolate them from the complex and the surrounding environment. $\mathrm{NH}_{2} \mathrm{O}$, which provides active aluminum atoms, was deposited on the silica shell. Finally, morin reacted with active aluminum atom to prepare the final multifunctional nanomaterial [2].

Calligraphy and painting identification is the basic history of calligraphy and painting research. The empirical means of traditional calligraphy and painting identification rely on intuition, which is very uncertain and easy to make. And because of ancient calligraphy and painting, even though we can collect samples for measurement, its specificity and accuracy are still difficult to determine time. Therefore, Bajju emphasizes that we should treat calligraphy and painting identification correctly and reasonably and recognize the uncertainty and uncertainty limitation of calligraphy and painting identification, and we should effectively use the research results of evaluation as methodology, not just pay attention to its conclusion, which is of great significance to the study of calligraphy and painting history [3]. By reviewing the development of Xu Bangda's research on traditional Chinese calligraphy and painting identification, Callery fully affirmed Xu Bangda's basic contribution to traditional Chinese painting and calligraphy identification and summarized Xu Bangda's methodology: artistic style comparison, style research, and demonstration and visual inspection and text combination research. Finally, the relationship between calligraphy and painting identification and art history is analyzed [4]. Throughout their masterpieces, there are many outstanding Chinese painters. The imitation, forgery, and ghost creation of ancient Chinese painting and calligraphy have brought many troubles and confusion to the appreciation and research of ancient Chinese painting and calligraphy. On the basis of certain theories and practices of calligraphy and painting identification, Chooto and Manaboot summed up eight basic methods for identification of ancient Chinese calligraphy and painting [5].

Based on the Raman measurement experiment, the microscale mechanical behavior of materials was studied from the response of material lattice structure to load. Combined with the experimental results of other scales, the multiscale mechanical properties and micromechanism of carbon nanotube fiber and film materials were systematically analyzed.

\section{Nanomaterial Preparation and Art Appraisal}

\subsection{Spectral Pretreatment Method}

2.1.1. Smoothing. Smoothing is usually used to avoid the influence of random noise on the spectrum, so it can make the sudden change in signal smoother and improve the signal to noise equipment, but spectral distortion may occur $[5,6]$. Generally, three compressor methods are used, namely, semiaverage method, moving average compressor method, and SG line compressor method. The semiaverage method is to divide the spectral data equally, using the central average of each segment instead of the original value of the equal segment [7]. The more data points in each class, the more serious the spectral distortion. To improve the averaging method, the fans of different cars overlap. The SG integration method is also called polynomial compressor method because of the same processing and signal distortion, so it is not necessary to grasp and maintain the peak and minimum positions of spectrum $[8,9]$.

The basic principle of SG smoothing is the polynomial $D$ "multiplication" of the measured points and the left and right sides of the measurement points. The fitting value of the center point is obtained from the measured point after 20 points, and the new value can be obtained by using 13 new polynomials in the new window. In the window, the data of equidistant points $n=2 m+1$ are matched with $k$-degree polynomial:

$$
\begin{aligned}
x_{j}^{i} & =a_{0}+a_{1} j+a_{2} j^{2}+\cdots+a_{k} j^{k}, \\
j & =-m,-m+1, \ldots, m-1, m ; i=1, \ldots, n .
\end{aligned}
$$

Formula (1) displays the position of data points in the overall measurement vector, and $j$ displays the position of data points on the left and right of the window. SG smoothing can adjust the window size and polynomial series number to get the desired smoothing effect. If the window width is too large, it will distort and the noise will not be eliminated if it is too small, so the appropriate window width is chosen $[10,11]$.

2.1.2. Derivative. The derivative algorithm can clear the outline of pectin and amplify the noise signal by eliminating the standard drift and change caused by the background color and other factors [12]. Spectrum's Judo generally adopts the SG interactive induction method and direct differentiation method. Direct precipitation method has large parameters in the spectral derivation of rare wavelength sampling points, but the SG interactive derivation method has no such problem in the spectral derivation of rare wavelength sampling points. The direct precipitation method is more suitable for high-resolution multiwavelength sampling. Since the spectra of bright spots are derived, different methods are directly used in this paper $[13,14]$. If the first derivative of the spectrum is obtained, the conversion error will be eliminated. By obtaining the second derivative spectrum, the rotation error of the spectrum can be eliminated. The derivation formula is as follows. 
The formula of the first derivative (FD) of direct difference is as follows:

$$
y^{\prime}=\frac{y_{(i+1)}-y_{i}}{\Delta \lambda} .
$$

The formula of the second derivative (SD) is as follows:

$$
y^{\prime \prime}=\frac{y_{(i+1)}-2 y_{i}+y_{(i-1)}}{\Delta \lambda^{2}}
$$

Among them, $y$ is the absorbance and $\Delta \lambda$ is the wavelength interval.

2.1.3. Light Scattering Correction. The spectral difference caused by scattering is greater than that caused by the internal components of the sample itself. The scattering degree is related to the wavelength, particle size, and refractive index of the sample, which mainly shows the conversion, rotation, quadratic, and high curve of the standard line. Scattering correction can effectively eliminate or reduce low light-level problems, reduce noise, and improve signal to noise. The commonly used methods are multivariable scattering correction and standard normal transformation [15].

The multideformation scattering correction can modify the various spectra due to scattering and obtain ideal spectra. The average spectrum of the set to be modified is the ideal spectrum. Each spectrum has an ideal spectrum and linear relationship, so the calculation based on the inclination of the line can be obtained by linear regression [16]. By the mathematical method, the scattering signal of the sample spectrum is separated from the internal component information of the sample, and the random variation is eliminated as much as possible. The calibration process is as follows:

(1) Calculate the average spectrum of the set to be corrected:

$$
\bar{A}=\sum_{i=1}^{n} \frac{A_{i}}{n} .
$$

(2) The regression coefficient $M_{I}$ and the regression constant $B_{i}$ of each spectrum were obtained by linear regression:

$$
A_{i}=m_{i} \bar{A}+b_{i}
$$

(3) After subtracting the regression constant from each original spectrum and dividing it by the regression coefficient, the multiple scattering correction is performed for the spectrum:

$$
A_{i(\mathrm{MSC})}=\frac{\left(A_{i}-b_{i}\right)}{m_{i}} .
$$

Formally, $A$ represents the $n \times p$ spectrum matrix to be modified, $n$ is the number of samples, and $p$ is the wavelength fraction of the spectrum. $A_{i}$ represents the spectrum of the second sample of $i$. The size of the interactive $m_{j}$ can reflect the inherent reflection effect of the sample, and the size of the slope reflects the uniformity of the sample. Adjusting the size of $m_{i}$ and $b_{i}$ can minimize the scattering signal of the spectrum $[17,18]$.

SNV (standard norm variate) assumes that the absorbance value of each wavelength point in each spectrum satisfies a specific distribution (such as a regular distribution). According to the difference of the family, each spectrum will be modified [19]. First, the mean value $\overline{X_{i}}$ and standard deviation of the sample spectrum is calculated to be corrected as $q$, and then, the mean value is subtracted from the sample spectrum to be corrected and divided by the standard deviation even if the original spectrum standard is normalized. It is calculated as follows:

$$
X_{i(\text { snv })}=\frac{X_{i}-\overline{X_{i}}}{\sigma_{i}},
$$

where $X_{i}$ represents the spectrum of the $i$-th sample.

2.2. Qualitative Analysis Methods. By comparing the spectra of known samples and unknown samples for qualitative analysis, due to the large overlap of absorption peaks in the broadband, it is difficult to judge the similarity with the naked eye, so a pattern recognition method is required. The pattern recognition method is to form a multidimensional space through the spectral information of the sample or the extracted characteristic data. Similar substances exist in similar positions in the space $[20,21]$. Therefore, the unknown range of the sample can be investigated based on this. At this time, there is no need to know the characteristic information of the sample. The spectral characteristics of the sample are analyzed. Commonly used pattern recognition methods include partial least squares, discriminative analysis, random forest, and extreme learning instruments [22].

2.2.1. Random Forest. Random Forest (RF) algorithm is a classification machine that contains multiple random decision trees that are essentially unrelated. The output result is the most output category of all christmas trees, and its generalization performance is excellent. The decision tree and the number of node division variables mtry in RF are two important parameters. In this article, the number of decision trees is optimized, and the number of node division variables is the basic value. The detailed process of the specific algorithm is as follows:

(1) $N$ crystal trees $C_{2}, C_{2}, \ldots, N$ training suits $S_{1}$ and $S_{2}$, corresponding to $\mathrm{CN}$; in order to randomly generate $\mathrm{SN}$, the start band method is used.

(2) Before selecting the attribute in each internal node, the segmentation attribute of the node is the $m$ attribute selected arbitrarily in the $M$ sample attributes, and the node is divided into the best segmentation method among the $m$ attributes (the value of $m$ 
generally does not change during the process, which is 0 and an integer between $M$ ).

(3) Each tree grows worthless, and each crystalline tree is used to distinguish the predicted sample $X$, and the corresponding categories $C_{2}(X), C_{2}(X)$, and $C N(X)$ are obtained.

(4) According to the principle followed by a few people, the category with the most output categories in all decision trees is regarded as the category of the prediction sample $X$.

(5) The principle of starting the trap method sampling includes $n$ different samples $\left(x_{1}, x_{2}, \ldots, x_{n}\right)$ in the set $S$. Each time the sample is changed, the number of extractions is assumed to be $n$. Second, after combining the extracted samples into the new set $S^{*}$, the probability that a specific sample $x_{i}(i=1,2, \ldots, n)$ is not included in $S^{*}$ is as follows:

$$
p=\left(1-\frac{1}{n}\right)^{n}
$$

When $n \longrightarrow \infty$, there are

$$
\lim _{n \longrightarrow \infty}=\lim _{n \longrightarrow \infty}\left(1-\frac{1}{n}\right)^{n}=e^{-1} \approx 0.368 .
$$

From the perspective of the total number of samples, the new set $S^{*}$ and the original set $S$ are both $n$, but due to the replacement of samples, the new set $S^{*}$ may contain the same samples repeatedly configured.

2.2.2. Extreme Learning Machine. Extreme Learning Machine (ELM) solves the shortcomings of slow training speed, complex parameter selection, and the easiest to cross the local neural network. It provides fast learning speed, more powerful generalization functions, and fewer adjustments, few advantages of media variables. ELM will randomly generate the connection weight value of the input layer and the hidden layer and the critical value of the hidden layer Nurem, so there is no need to adjust. The number of hidden stacked neurons and their activation functions (only nonlinear, nondiscriminatory, and discontinuous functions) and the only optimal solution $\beta$ can be obtained. The detailed process of the specific algorithm is as follows:

(1) Set the number of hidden layer neurons and automatically generate the connection weight $w$ between the input layer and the hidden layer and the hidden layer neuron value $b$.

(2) Set the activation function of the hidden layer neuron and calculate the output matrix $H$ of the hidden layer.

(3) Calculate the weight of the output layer $\hat{\beta}: \hat{\beta}=H+T^{\prime}$.

\subsection{Physical Preparation Methods of Nanomaterials}

2.3.1. Mechanical Law. Mechanical methods include mechanical ball, mechanical crushing, and high gravity technology. Mechanical ball method does not need external heat supply. Through ball sealing, due to the surface reaction between materials, large particles can be changed into small particles to obtain nanomaterials. Mechanical comminution adopts many methods, such as mechanical comminution of superfine micropowder and electric spark explosion, to directly crush raw materials into ultrafine powder, which is especially suitable for manufacturing ultrafine powder of finished products. High gravity technology is the high-speed rotation of high-gravity rotation layer. Centrifugal acceleration hundreds of times the acceleration of gravity greatly improves the material transmission and fine mixing between each other to produce nanomaterials.

2.3.2. Gas Phase Method. Meteorological methods include the evaporation condensation method, solution evaporation method, and plastic deformation method. Evaporation and condensation methods are to gasify raw materials or form plasma and to achieve a supersaturation state by resisting heating, high-frequency induction, plasma, laser, electron beam, and arc induction in vacuum or inactive gas and gas media. In order to form high-purity nanomaterials, the condensation solution evaporation method in the media is to make the solvent into small bells and then quickly evaporate them to minimize the separation of components. It can be treated by spray drying, spray pyrolysis, or freeze drying. Severe plastic deformation is a function of quasistatic pressure. In this case, the size of the material is slightly adjusted to nanolevel after plastic deformation.

2.3.3. Magnetron Sputtering and Plasma. Stamping technology uses high-energy particles to exchange energy or motion. Atoms or molecules on the surface of the target material fly to the surface of the target material and adhere to the substrate to form nanomaterials. The formation of the compound is not easy to change. At present, spring technology has been greatly developed, which generally uses carbide, DC magnetic field, high-frequency magnetic field, ion beam pusher, and electron tetranuclear tube resonanceassisted reactive magnetic field. The plasma method uses DC discharge in inactive or reactive atmosphere to ionize the gas and produce high-temperature plasma. The steam is cooled to form ultrafine particles by combining and evaporating the raw material solution. High ionic temperature can produce refractory metals or compounds with high purity. In an inactive atmosphere, almost all metal nanomaterials can be produced by the plasma method.

\subsection{Chemical Preparation of Nanomaterials}

2.4.1. Sol-Gel Method. The chemical engineering of gelation is to disperse raw materials in solvents and then generate active simulators through hydrolysis and decomposition. 
2.4.2. Ionic Liquid Method. As a special organic solvent, ionic liquids have inherent physical and chemical properties such as high viscosity, high ionic conductivity, high thermal stability, low toxicity, excellent fluidity, and wide liquid temperature range. At a higher temperature, ionic liquid is still of low volatility, which is difficult to cause environmental pollution. It is a kind of green solvent, and ionic liquid is a good medium to synthesize different nanostructures.

2.4.3. Solvothermal Method. Solvothermal method is an effective method to realize controllable synthesis and manufacture of nanomaterials by heating the reaction system composed of various solvents in a closed reactor (such as automatic clay), so as to form an effective method in hightemperature and high-pressure environment.

2.4.4. Microemulsion Method. Nanoparticle microemulsion manufacturing is a newly developed research area with the advantages of being close to small particle size and monodisperse system.

\section{Nanomaterial Preparation and Art Appraisal Test Design}

3.1. Experimental Materials and Testing Instruments. S powder (purity 99.99\%) and nanopowder (purity 99.99\%) were used as raw materials. The sample collection substrate is an $n$-type monocrystalline silicon wafer (111) ground on one side. The substrate was washed with acetone and water. In the experiment, as a shielding gas, high-purity Ar gas is needed.

The surface morphology of samples on silicon wafers was detected and analyzed by injection electron microscopy (SEM, s-4800) equipped with energy spectrum X-ray (EDX). The structure of the sample was measured and analyzed by $\mathrm{X}$-ray system (CRD, Rigaku Ultima IV, $\mathrm{Cu}-\mathrm{K} \alpha$ radiation). The Raman spectrum of the sample was detected and analyzed by LabRAM HR of $532 \mathrm{~nm}$ laser line. The luminescence (PL) spectrum was obtained by the detection of $325 \mathrm{~nm} \mathrm{He}$-Cd laser at room temperature.

3.2. $\mathrm{SiO}_{2}$ Nanocrystals and Amorphous Nanowires. The growth of nanostructures is carried out in a traditional horizontal quartz tube. In the experiment, Si nanopowder and $s$ powder (purity 99.99\%) were mixed uniformly in the ratio of $1: 1$, and the quartz tube was placed in the hightemperature region of horizontal tube. The $n$-type Si (111) sheet is located $10-20 \mathrm{~cm}$ from the reaction source, and the $\mathrm{Si}$ sheet is relatively low in commercial use. The impurity gas of penicillin is removed as much as possible through the protective gas Ar for 20 minutes before the temperature rises. After that, it was heated to $1000^{\circ} \mathrm{C}$ in 2 hours at the rate of $20 \mathrm{ml} / \mathrm{min}$ under a certain flow of Ar. After the reaction, the flow of Ar stopped. At the end of the experiment, the samples were taken out, and white sponge and other substances were found on the surface of silica gel.
3.3. Art Identification Experiment. German Bruker Fouriertransform near-infrared Raman spectrometer, Japanese 1SM-6390 scanning electron microscope, and German Bruker infrared absorption instrument were used as experimental instruments. By comparing the Raman spectra of the pigments studied with those of various monochromatic pigments, the formation and content of the studied pigments can be roughly obtained according to the position and intensity of the Raman peaks. The reflectance of various monochromatic pigments should be considered when comparing the Raman spectra of pigments with those of various monochromatic pigments.

\section{Analysis of Nanomaterial Preparation and Art Appraisal Test Results}

4.1. Preparation and Analysis of Nanomaterials. The experimental results show that a large number of nanowires and nanocrystals appear at $950^{\circ} \mathrm{C}$ due to the uneven growth. The diameter of the line is between 100 and $400 \mathrm{~nm}$, and the length is $0.8-3 \mu M$. The grain size is about $200-460 \mathrm{~nm}$. The EDX spectra show that the nanowires and nanocrystals are composed of $\mathrm{Si}$ and $\mathrm{O}$ elements. The ratio of silicon to oxygen is close to $1: 2$, and a small amount of extraoxygen may come from the test environment of EDX technology. The morphology of nanowires is grown at $950^{\circ} \mathrm{C}, 960^{\circ} \mathrm{C}$, and $970^{\circ} \mathrm{C}$, respectively. It is obvious that the diameter of nanowires increases and the length decreases with the increase in growth temperature. Nanoparticles are distributed on the surface of the whole Si substrate, and their sizes are between $100 \mathrm{~nm}$ and $380 \mathrm{~nm}$. The three peaks at $21.5^{\circ} \mathrm{C}$, $23.9^{\circ} \mathrm{C}$, and $26.7^{\circ} \mathrm{C}$ positions can be indexed as crystalline $\mathrm{SiO}_{2}$, which matches the diffraction pattern of rhombic $\mathrm{SiO}_{2}$ (PDF no. 03-0419). The structure of such "rhombic $\mathrm{SiO}_{2}$ " nanocrystals is commonly referred to as $\alpha$-quartz. In addition to the crystalline phase, there is a wide peak of $\mathrm{SiO}_{2}$ between $12.3^{\circ} \mathrm{C}$ and $32.1^{\circ} \mathrm{C}$.

The Raman spectra of $\mathrm{SiO}_{2}$ nanowires and nanoparticles are shown in Figure 1.

There is a unique peak at $521 \mathrm{~cm}^{-1}$, corresponding to the Si substrate. It has been reported that the signal of Si band in amorphous $\mathrm{Si}$, Si nanocrystals, or Si nanowires is generally in the range of $480-510.5 \mathrm{~cm}^{-1}$. However, there is no peak in this range, which indicates that the product is likely to be oxidized.

The EDX spectrum of nanowires with large area is shown in Figure 2.

The test found that the content ratio of $\mathrm{Si}$ to $\mathrm{O}$ is close to $1: 2$. The silicon-oxygen $1: 2$ ratio will be affected by the silicon substrate, leading to inaccurate thoughts about the silicon-oxygen content ratio. But through the Raman test and through the above TEM results, except for the $\mathrm{SiO}_{2}$ amorphous nanowires, the nanoparticles are essentially polycrystalline. The XRD results show that the synthesized crystal is a rhombohedral structure of $\mathrm{SiO}_{2}$. Therefore, it can be reasonably concluded that the products grown on $\mathrm{Si}$ substrates are $\mathrm{SiO}_{2}$ amorphous nanowires and nanocrystals. Amorphous/nanocrystalline Si nanoparticles do not appear in the product. 


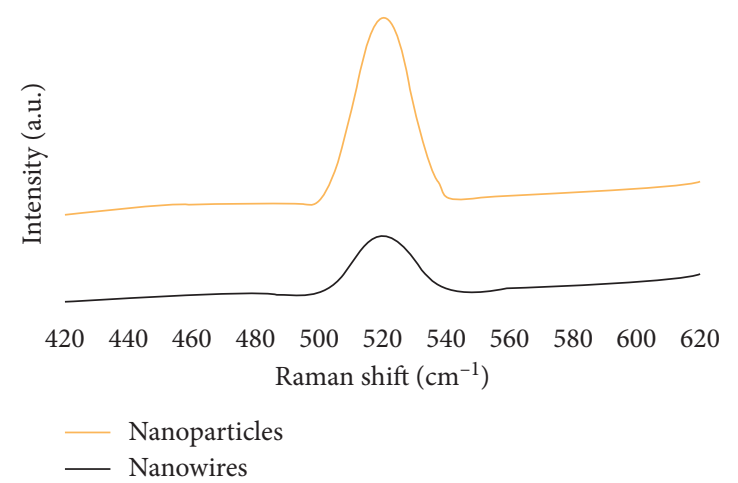

FIGURE 1: Raman spectrum of synthesized $\mathrm{SiO}_{2}$ nanowires and nanoparticles.

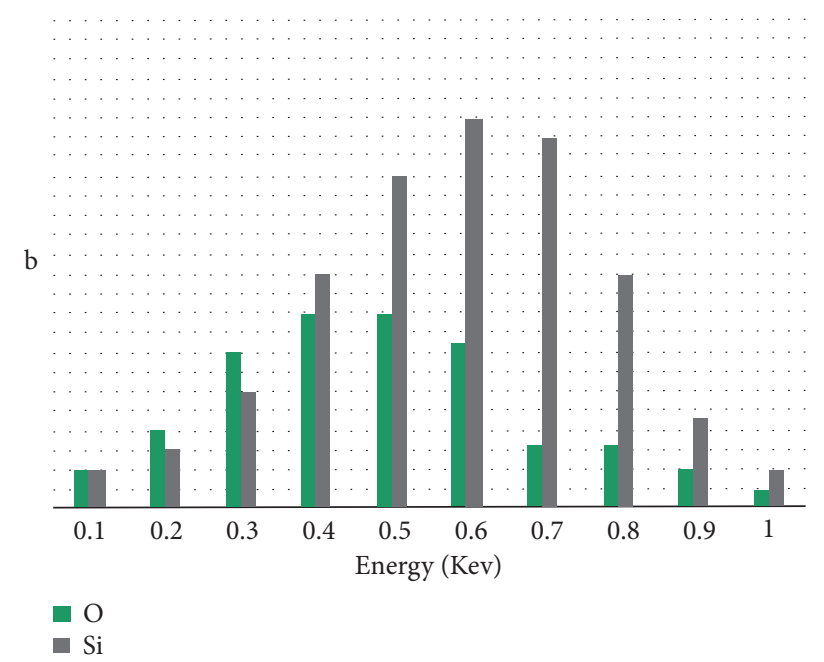

FIgURE 2: EDX spectrum of the synthesized nanowire.

$S$ powder reacts with $\mathrm{Si}$ wafers at a lower temperature (about $900^{\circ} \mathrm{C}$ ) to form silicon sulfide (SiS). When the temperature rises above $1000^{\circ} \mathrm{C}$, Si nanowires are precipitated from $\mathrm{SiS}$ and grow into nanowires in a certain direction. SiS is located at the tip of the Si nanowire. It can be said that $\mathrm{SiS}$, as a nucleation center, plays an important role in assisting the growth process. In our experimental study, Si powder and $S$ powder were thermally evaporated at a lower temperature under argon flow to prepare $\mathrm{SiO}_{2} \mathrm{ANW}$ and NC. The Si/O content ratio at different positions of the nanowires was measured by EDX. Since a Si substrate is used, the Si content is usually relatively high. From the test in the figure, it is found that the $\mathrm{O}$ content at the top of the nanowire is slightly higher than the middle position. According to the SEM results, the length of the nanowires increases as the growth temperature decreases. This may indicate that the nanowires are growing in the radial direction. Similar to the previous report the nucleation center is located at the tip of the nanowire. The particle generation and oxidation during the reaction is accompanied by an increase in O. Therefore, the high oxygen content on the top of the nanowire corresponds to the top growth mechanism of the nanowire.
4.2. Art Appraisal Analysis under Spectroscopic Technology. The ten papers in the experiment were all manufactured in different places or by different manufacturers. During the experiment, visible Raman (wavelength is $514 \mathrm{~nm}$ ) is first used to detect these samples. But most of the samples have too strong fluorescence, and some samples are very weak even with peaks. Therefore, the ultraviolet-visible Raman spectrometer was used to do the laser fluorescence of these samples, and the excitation wavelength was $514 \mathrm{~nm}$. The fluorescence peaks are analyzed of the first five samples, as shown in Figure 3.

It is obvious from Figure 3 that the fluorescence peaks of the samples are not the same. Sample 1 and sample 4 both have a fluorescence peak at $630 \mathrm{~nm}$, but sample 1 has a small shoulder at $673 \mathrm{~nm}$. The fluorescence peaks of sample 2 and sample 4 are at $608 \mathrm{~nm}$ and $646 \mathrm{~nm}$, respectively. The most special sample is No. 3, which has three very obvious fluorescence peaks, namely, $630 \mathrm{~nm}, 669 \mathrm{~nm}$, and $760 \mathrm{~nm}$. Their corresponding peak positions are shown in Table 1.

The fluorescence spectra of samples 6-10 are shown in Figure 4 .

The fluorescence peaks of sample 6 and sample 7 are the same, the fluorescence peak of sample 9 at $640 \mathrm{~nm}$ is at 


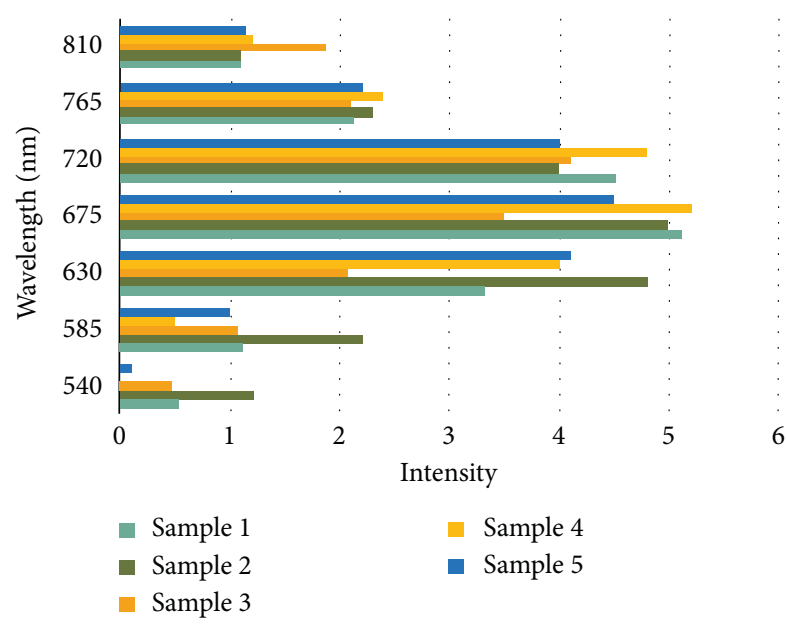

FIGURE 3: Laser fluorescence of samples 1-5 paper.

TABLE 1: Mahalanobis distance from inkpad on different carriers to its actual category.

\begin{tabular}{lccc}
\hline Sample & \multicolumn{3}{c}{ Mahalanobis distance } \\
\hline 1 & 0.90 & 0.97 & 0.83 \\
2 & 1.01 & 1.00 & 0.79 \\
3 & 1.13 & 0.77 & 1.12 \\
4 & 0.47 & 0.39 & 0.45 \\
5 & 0.41 & 0.59 & 0.29 \\
\hline
\end{tabular}

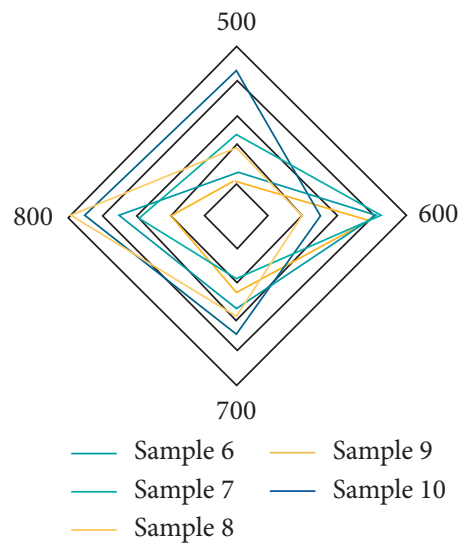

FIGURE 4: Laser fluorescence of samples 6-10.

$623 \mathrm{~nm}$, and there is a shoulder peak around 650. The peak of sample no. 8 is at $650 \mathrm{~nm}$. The fluorescence peaks of sample no. 10 are $589 \mathrm{~nm}, 623 \mathrm{~nm}$, and $671 \mathrm{~nm}$, respectively.

By observing the scanning electron micrograph of the rice paper, we can analyze the general ratio of leather and grass in the paper from the surface structure of the rice paper or the number of long and short fibers in them and further use this method to identify the type of rice paper. By observing the scanning electron micrograph of rice paper, we can roughly distinguish the differences in the manufacturing process of different manufacturers from the size and structure of the fiber. This also provides a way to identify different origins or manufacturers of rice paper.

\section{Conclusions}

Spectroscopy technology is widely used in the fields of medicine, cultural heritage, gemstone identification, and forensic medicine. Through the destruction analysis and research of cultural relic samples, the identification, dating, restoration, and preservation of cultural relics can be made safer and more trustworthy. Analysis and research can understand the different points contained in gems in different places and provide scientific basis for identifying and evaluating gems. The use of spectroscopy technology can achieve recognition accuracy and avoid damage to calligraphy and images. Therefore, with the development of modern technology, spectroscopic technology will become a necessary means of identification.

In this study, thermal evaporation was used to produce nano- $\mathrm{SiO}_{2}$ materials with different shapes and structures. The basic structure, photoelectric properties, and manufacturing process of the surface were studied and analyzed. Through experiments, it is found that the structure and morphology of the samples obtained at different adhesion temperatures and increased pressures are different, and the diameter and length of the samples are affected by the growth temperature. In addition, the optical properties of nano- $\mathrm{SiO}_{2}$ with different morphologies and structures are also very different. In these two aspects, in order to change the size 3 gap, the size or the band gap of other materials can be changed to improve the complexity and function of the nanostructure. Therefore, the research on other forms of nano- $\mathrm{SiO}_{2}$ will help to develop its characteristics, which will greatly help the better application of electronic equipment and new materials.

In this study, rice paper from different manufacturers was selected. Due to its strong fluorescence, it is not only difficult to form a visible Raman spectrum, but the near-infrared signal is also weak. In this case, in order to perform laser fluorescence, the wavelength of $514 \mathrm{~nm}$ is used here, and finally, good experimental results are obtained. The rice paper of other manufacturers is made of other raw materials or the same kind of raw materials, but the ratio is different, and the final fluorescence spectrum is also different, which can distinguish the paper made by other manufacturers and provide a method for the identification of calligraphy and painting in the future.

\section{Data Availability}

The data in this article are actually available.

\section{Disclosure}

We confirm that the content of the manuscript has not been published or submitted for publication elsewhere.

\section{Conflicts of Interest}

The author declares that there are no conflicts of interest in our paper. 


\section{Acknowledgments}

This work was supported by the Hubei Institute of Fine Arts.

\section{References}

[1] A. Gonciarz, M. Żuber, and J. Zwodziak, "Front cover: spectrochemical properties and solvatochromism of tetradentate schiff base complex with nickel: calculations and experiments (ChemistryOpen 9/2018)," ChemistryOpen, vol. 7, no. 9, p. 669, 2018.

[2] P. Duan, B. Liu, C. L. M. Morais et al., "4-Nonylphenol effects on rat testis and sertoli cells determined by spectrochemical techniques coupled with chemometric analysis," Chemosphere, vol. 218, pp. 64-75, 2018.

[3] G. D. Bajju, G. Devi, and A. S. Ashu, "Spectrochemical and biological evaluation of axially substituted zirconium (IV) meso-tetra (4-methoxyphenyl)porphyrins," Russian Journal of Inorganic Chemistry, vol. 64, no. 6, pp. 734-741, 2019.

[4] E. L. Callery, C. L. M. Morais, M. Paraskevaidi et al., "New approach to investigate common variable immunodeficiency patients using spectrochemical analysis of blood," Scientific Reports, vol. 9, no. 1, p. 7239, 2019.

[5] P. Chooto and S. Manaboot, "Electrochemical, spectrochemical and quantum chemical studies on dimedone as corrosion inhibitor for copper in acetonitrile," Journal of Scientific Research and Reports, vol. 15, no. 2, pp. 1-13, 2017.

[6] A. S. Zakuskin, A. M. Popov, N. B. Zorov, and T. A. Labutin, "Confinement of laser plasma by shock waves for increasing signal intensity in spectrochemical determination of trace elements in ores," Technical Physics Letters, vol. 44, no. 1, pp. 73-76, 2018.

[7] K. Li, C. F. Ma, Y. Ling, M. Li, Q. Gao, and W. J. Luo, “Acid/ base bifunctional carbonaceous nanomaterial with large surface area: preparation, characterization, and adsorption properties for cationic and anionic compounds," Materials Chemistry and Physics, vol. 162, no. 8, pp. 149-161, 2015.

[8] J. Kamalakkannan, V. L. Chandraboss, B. Karthikeyan, and S. Senthilvelan, "Preparation and characterization of TiInVO6-nanomaterial using precipitation method and its multi applications," Journal of Materials Science: Materials in Electronics, vol. 27, no. 3, pp. 2488-2503, 2016.

[9] M. Hasanzadeh, S. A. Nahar, N. Shadjou, and A. Mokhtarzadeh, "Immobilization of proline dehydrogenase on functionalized silica mesoporous nanomaterial towards preparation of a novel thermostable enzyme biosensor," Journal of Nanoscience and Nanotechnology, vol. 18, no. 11, pp. 7786-7796, 2018.

[10] S. Akai, N. K. Kumagai, Y. Sasaki et al., "Development of automatic TEM/SEM specimen preparation instrument for nanomaterial dispersed in liquid," Microscopy and Microanalysis, vol. 25, no. S2, pp. 774-775, 2019.

[11] Q. Kikuchi, Y. Wang, J. Xiao, C. Chen, and H. Fan, "Preparation and characterization of magnetic nanomaterial and its application for removal of polycyclic aromatic hydrocarbons," Journal of Hazardous Materials, vol. 371, pp. 323-331, 2019.

[12] A. Lisowska-Oleksiak, B. Wicikowska, A. Nowak et al., "Preparation and characterization of nanomaterial consisting of silica aerogel \& carbon tested as an electrode in nonaqueous media containing lithium salt," International Journal of Electrochemical Ence, vol. 11, no. 3, pp. 1997-2007, 2016.

[13] Y. B. Tao, C. H. Lin, and Y. L. He, "Preparation and thermal properties characterization of carbonate salt/carbon nanomaterial composite phase change material," Energy Conversion and Management, vol. 97, pp. 103-110, 2015.

[14] A. Dash and R. Chakravarty, "Nanomaterial-based adsorbent: promises, opportunities, and challenges to develop column chromatography radionuclide generators for nuclear medicine," Separation \& Purification Reviews, vol. 46, no. 2, pp. 91-107, 2016.

[15] H. Gao, "The value and a preliminary study of the integration of traditional Chinese painting and calligraphy \& modern and contemporary art in primary school art teaching," International Education Studies, vol. 12, no. 6, p. 75, 2019.

[16] V. Y. Davydov, A. N. Smirnov, I. N. Goncharuk et al., "Raman spectroscopy as a tool for characterization of strained hexagonal GaN/AlxGa1-xN superlattices," Physica Status Solidi, vol. 234, no. 3, pp. 975-979, 2015.

[17] P. Crow, J. S. Uff, J. A. Farmer et al., "The use of Raman spectroscopy to identify and characterize transitional cell carcinoma in vitro," BJU International, vol. 93, no. 9, pp. 1232-1236, 2015.

[18] A. Akemi Ooka and R. L. Garrell, "Surface-enhanced Raman spectroscopy of DOPA-containing peptides related to adhesive protein of marine mussel, Mytilus edulis," Biopolymers, vol. 57, no. 2, pp. 92-102, 2015.

[19] G. H. Smudde and P. C. Stair, "The oxidation of Mo (100) studied by XPS and surface Raman spectroscopy: the onset of $\mathrm{MoO}_{2}$ formation and the formation of surface polymolybdate," Surface Science, vol. 317, no. 1-2, pp. 65-72, 2016.

[20] F. Collard, B. Gilbert, G. Eppe, K. Das, and E. Parmentier, "Detection of anthropogenic particles in fish stomachs: an isolation method adapted to identification by Raman spectroscopy," Archives of Environmental Contamination and Toxicology, vol. 69, no. 3, pp. 331-339, 2015.

[21] N. Q. Liem, D. X. Thanh, V. X. Quang et al., "Detection of lattice match between $\mathrm{ZnSxSe1-x}$ epilayers and GaAs substrates by polarisation Raman spectroscopy," Physica Status Solidi, vol. 229, no. 1, pp. 47-51, 2015.

[22] S. A. Overman and G. J. Thomas, "Structural studies of viruses by Raman spectroscopy. Novel vibrational assignments for proteins from Raman spectra of viruses," Journal of Raman Spectroscopy, vol. 29, no. 1, pp. 23-29, 2015. 